\title{
THE ROLE OF THE TECTAL COLUMN IN FACILITATION OF AMPHIBIAN PREY-CATCHING BEHAVIOR: A NEURAL MODEL ${ }^{1}$
}

\author{
ROLANDO LARA, ${ }^{2}$ MICHAEL A. ARBIB, ${ }^{3}$ AND ANDREW S. CROMARTY \\ Center for Systems Neuroscience and Computer and Information Science Department, University of Massachusetts, \\ Amherst, Massachusetts 01003
}

Received February 20, 1981; Revised December 4, 1981; Accepted December 12, 1981

\begin{abstract}
We used computer analysis of differential equations to study the properties of a family of models of a unit of neural circuitry in the amphibian tectum, the tectal column. Computer experiments were used to discriminate among various hypotheses and to suggest new experiments. Particular attention was paid to physiological data on facilitation of amphibian prey-catching behavior which led us to model the facilitation in terms of dynamic activity in the tectal column rather than in terms of synaptic modification.
\end{abstract}

In the present paper, we propose a mathematical model of the amphibian tectal column to account for the facilitatory effects of prey-catching activity when prey stimuli are presented. The model is based on anatomical and physiological studies of this region and behavioral results observed in facilitation of prey-catching activity. The structure of the tectum shares many properties with other brain regions, which allow us to supplement the available tectal data. The model proposed is flexible and offers several alternatives that could be tested experimentally to constrain the number of possible mechanisms proposed. The current model uses simple differential equations to represent the time course of the membrane voltage of the cell and uses threshold functions to compute the corresponding action potentials. The model was simulated on a Digital Equipment Corp. VAX 11/780.

Our work is informed by the following features that brain regions share in their sensorimotor processing of information:

(1) In many systems, afferents are processed initially by a synaptic complex, a glomerulus comprising specific connections between axonal and dendritic terminals which may be interconnected, enclosed in glial membranes, or otherwise set apart. This synaptic complex has been described in many brain regions (Shepherd, 1970, 1974; Szentágothai, 1970; Székely and Lázár, 1976), such

\footnotetext{
'The research reported in this paper was supported in part by National Institutes of Health Grant NS14971. We thank David Ingle and Peter Ewert for valuable discussions of the experimental data and Donald House for his help with the computer graphics.

${ }^{2}$ Present address: Universidad Nacional Autonoma de Mexico, Centro de Investigaciones en Fisiologia Celular, A.P. 70-600, Mexico 20, D.F., Mexico.

${ }^{3}$ To whom correspondence should be addressed.
}

as the olfactory bulb, the thalamus, the cerebral cortex, the cerebellum, the spinal cord, and the amphibian optic tectum. There is a strong indication that a glomerulus functions to some extent as a functional unit.

(2) Afferent information can be processed in both a lateral and a columnar organization, depending on the vertical or lateral dispersion of the information received. Vertical processing of information has been found (Shepherd, 1974; Szentágothai and Arbib, 1974; Mountcastle, 1957; Hubel and Wiesel, 1963) in the olfactory bulb, in many regions of the cortex (visual, auditory, and somatosensory), and in the optic tectum of amphibia. The elementary unit of vertical processing is held to be the column. From an anatomical-functional point of view, it has been defined (Mountcastle, 1978; Szentágothai and Arbib, 1974) as an integrative unit of the neural tissue of minimal size that, on the basis of internal connectivity, still could be considered the unitary neuronal machine for elementary tasks of information processing, since it is composed of neurons activated by stimulation of the same single class of peripheral receptors and has almost identical peripheral fields. Lateral processing has been suggested as the dominant mode in some regions of the thalamus, hippocampus, olfactory cortex, and cerebellum. Some of these regions have been associated with the processing of sequential information.

(3) Sensorimotor processing involves a complex interaction of excitation and inhibition. In many regions of the nervous system, circuits have been described which provide for an initial excitation followed by a long lasting inhibition (Shepherd, 1974; Szentágothai and Arbib, 1974; Purpura, 1970). These include the spinal cord, cerebellum, olfactory bulb, olfactory cortex, hippocampus, thalamus, and cerebral cortex. Some of these regions 
also exhibit rebound excitation due mainly to two mechanisms: one is through recurrent axons which may produce long lasting depolarization in their respective cells as has been suggested to occur in the olfactory bulb (Shepherd, 1970), and probably the amphibian optic tectum (Székely and Lázár, 1976); the other is the product of reverberatory circuits such as those involving the cerebellum and reticular formation (Eccles, 1973), the hippocampus and the septum (Raisman et al., 1965), and the thalamus and the cortex (Singer, 1977). All of these processes are mediated by excitation, re-excitation, and inhibition.

We now incorporate these features in our analysis of tectal mechanisms in the facilitation of prey-catching behavior.

\section{Behavioral and physiological studies related to facilitation of prey-catching behavior}

David Ingle (1973, 1975) showed that a worm-like stimulus of $5^{\circ}$ presented for an interval of $0.3 \mathrm{sec}$ and moved $5^{\circ}$ of visual angle in a specific area of the retinal field of frogs does not induce prey-catching behavior, but if the same stimulus is presented again $2.3 \mathrm{sec}$ later, the likelihood of snapping was increased to $75 \%$. Moreover, this facilitation effect was restricted to the area previously stimulated, because re-presentation of the stimulus in a different region does not produce prey-catching behavior. These results indicate that the facilitatory effect involves little lateral spread of information, with the input processed in a predominantly vertical way. In the present paper, we shall analyze this vertical processing in terms of a posited anatomical column for optic tectum. Arbib and I ara (1982) consider the extension to an array of columns with local interaction.

Ingle (1975), trying to correlate the action of tectal cells with the observed behavioral results, found two cells whose temporal response could be related to the observed behavioral facts. He studied the activity of these cells, presenting as a stimulus a small square $2^{\circ}$ in size for an interval of $0.5 \mathrm{sec}$. One of these cells gave an initial response when the stimulus was shown, then a period of silence, and finally a slow steady discharge that lasted from 3 to $6 \mathrm{sec}$. The second type of neuron did not respond when the stimulus was present, but gave a short delayed burst (see Fig. $2 d$ ). Moreover, Ingle has shown that these cells are located more easily after pretectum ablation, which suggests that these cells may play an important role in prey-catching facilitation and normally are controlled by the inhibitory effect of the thalamuspretectum. Given anatomical studies of the tectum which show that cells send recurrent axons to their dendrites and given Ingle's data on the behavior of the tectal cells which show a rebound excitation in the absence of stimulation, we have modeled the present facilitation phenomenon by recurrent excitation and inhibition rather than in terms of synaptic facilitation.

\section{Anatomy of the tectum}

Székely and Lázár (1976) have classified the tectal cells on the basis of their shape, dendritic and axonal arborization, and the types of interactions with other cells. We will describe only those tectal cells that we have consid- ered in our model. The other tectal cells described by these authors were not considered in this model either because they are hard to find in the tectum (as is the case for bipolar, amacrine, and ganglionic cells) or because the anatomical literature has not established with which other tectal cells they synapse (as is the case for the second type of large pear cell). It must be stressed, however, that the formulation of the present model has involved a number of arbitrary choices that must be studied comparatively in the light of present and future experimental results. Moreover, the study of models based on alternative choices is an urgent topic for future theoretical research.

The tectal cells considered in our model (Fig. 1) are described below.

Large pear-shaped cell (LP). These cells are located in layer 6; they have a long apical dendrite that projects toward the surface and arborizes in a most variable manner. These arborizations are the main recipients of the optic terminals. The axon originates from the dendrites and takes an upward course close to the dendritic arbor of the parent cell.

Large pyramidal cell (PY). PY cells are located in layer 6 and have wide dendritic tree; these cells are regarded as the efferent elements of the tectum in our model. (The present model does not take into consideration the less common tectal-ganglion efferents.)

Small pear-shaped cells (SP). SP cells are located in layer 8; these cells, like the LP cells, have a large apical

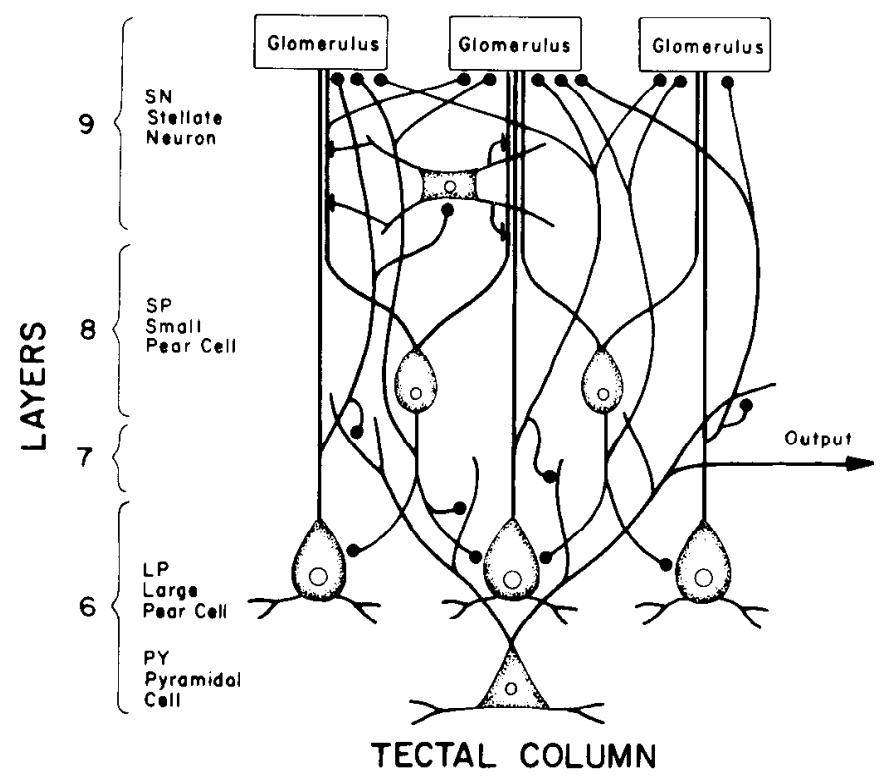

Figure 1. Neurons and synaptology of the model of the tectal column. The numbers at the left indicate the different tectal layers. The glomerulus is constituted by the LP and SP dendrites and recurrent axons as well as by optic and diencephalic terminals. The LP excites the PY, the SN, and the glomerulus and is inhibited by the SN (of which only one of the two in our model of the column is shown). The SP excites the LP and PY cells and it sends recurrent axons to the glomerulus; it is inhibited by the SN. The SN is excited by LP neurons and diencephalic fibers and it inhibits the LP and SP cells. The PY is activated by the LP, SP, and optic fibers and is the efferent neuron of the tectum. 
dendrite that projects toward the surface but with a different arborization. Some of these neurons have very short axons terminating in a moderately arborizing formation next to the parent perikaryon. In other cases, the axon descends into layer 6 and terminates among the cells of this layer. In the third type, the axon descends for a while and then, with a sharp loop, turns back and terminates in the lower part of layer 9 . In the present model, we have considered only the last type.

Stellate cells $(S N)$. These cells are located in layer 9 and have many axons originating from the dendrites. Axosomatic-somatodendritic synapses have been found on these cells, with axons coming both from the LP cells and diencephalic terminals and the dendrites of possibly LP and SP neurons.

The anatomical studies of the tectum indicate that this structure has mostly a vertical orientation, with the cells having recurrent axons and a small lateral spread of information. This led to the suggestion that the tectum processes the optic information in a vertical way by means of functional units comprising several cells. In the next section, on the basis of anatomical, behavioral, and physiological studies, we postulate the synaptology of the functional unit of the tectum, the tectal column. Our model shows how tectal activity can last for several seconds after the stimulus has disappeared through a combination of excitatory and inhibitory interactions.

\section{Model of the Tectal Column}

General structure. We have considered the tectum as comprising functional units, the tectal columns, as described by Székely and Lázár (1976). The lateral extension of each column is determined by the arborization of the dendrites of the pyramidal cell, the main efferent cell in the tectum. The number of cells in each column was chosen in proportion to the number of each cell type relative to the number of pyramidal cells. For this reason, each column has one pyramidal cell, three large pearshaped cells, two small pear-shaped cells, and two stellate interneurons (of which only one is shown in the figure). Each of these cells has a receptive field and an effector field, the size of which is dependent on the degrees of dendritic and axonal arborization, respectively. The general configuration of the column is shown in Figure 1. The mathematical description of this system as a set of simple differential equations (linear dynamics with step or threshold output functions) is provided in the appendix. The interest of the model is not in the individual neurons but rather in the experiments on the effects of changes in patterns of connectivity.

Synaptology and function of the tectal column. The optic afferents from retinal ganglion cells innervate the tectum mainly at the tectal glomerulus, types 1 and 2 in the superficial zone of layer 9 and types 3 and 4 in the deeper section of this layer. (In this model, we have considered only types 1 and 2; we do not model retinal processes, simply assuring in our simulation that relevant input is provided to each tectal neuron.) The glomerulus, besides the optic fibers, is composed of the dendrites and recurrent axons of LP and SP cells and fibers of diencephalic origin; optic afferents, internal axons of the tectum, and diencephalic fibers are all presynaptic with respect to the dendrites. The LP and SP dendrites can have dendrodendritic synapses in a nonreciprocal fashion. For comparison, note that the activity of the olfactory bulb shows long lasting oscillatory behavior after the stimulus has disappeared. This behavior led to the postulate that the stimulus, in combination with the recurrent activity of some cells in this structure, produces a long lasting depolarization in the glomerulus, which, in combination with the inhibitory activity of the mitral cells, produces the oscillatory response (Shepherd, 1970, 1974). We have proposed, similarly, that the state of excitation of the glomerulus in the optic tectum can be maintained for a long period after the stimulus has disappeared. This state of activity is maintained by the excitatory activity of both the dendrodendritic interactions and recurrent axons of LP and SP cells. We suggest this hypothesis, as did Székely and Lázár (1976), based on the fact that the optic input goes directly to the glomerulus which also receives the dendrites of the intratectal cells. An inhibitory effect at this level would seem to be an unlikely means of sending sensory information to the tectal column. In this way, the glomerulus may act as a functional unit in the sensorimotor processing of information.

In some regions of the nervous system, physiological study has shown (Shepherd, 1974; Purpura, 1970; Eccles, 1973; Kandel et al., 1961; Kandel and Spencer, 1961) that, after an EPSP produced by a given stimulus, there usually is a subsequent long lasting hyperpolarization. This inhibition can be obtained through feedback inhibition in, for example, the spinal cord, thalamus, cerebellum, cortex, and hippocampus or can be due to dendrodendritic inhibition as in the olfactory bulb, thalamus, retina, and possibly the cortex. In the present model, we propose that the stellate cell inhibits the activity of the tectal column through feedback inhibition in the following way.

When the glomerulus has been stimulated, it produces a long lasting depolarization that travels by the apical dendrite to the soma of LP and SP cells; if the excitation is strong enough, it may produce a neural response. When the LP cell is activated, it excites a stellate neuron, which recurrently inhibits the LP excitation. Moreover, the inhibitory effect of the SN neuron can lead to lateral inhibition or local control of the state of excitation of the column. We have postulated that the inhibitory effect of these cells is dependent on their state of excitation. This postulate is based on the fact that the peculiar synaptic organization of these cells allows it to exert both local and global control of the column activity. This control is obtained locally through complex units of axodendritic or axosomatic afferents and somatodendritic or dendrodendritic synapses that can function below action potential threshold; general control will be exercised if the level of afferent activity is sufficient to drive the stellate cell to discharge an action potential, silencing a broad area of the tectal column (Székely and Lázár, 1976).

At the same time, the recurrent axons of the LP cells maintain both the level of activity of their column's glomeruli in a vertical manner and the state of excitation of neighboring glomeruli through axon collaterals, thus spreading the excitation laterally across the tectum. This architecture permits us to simulate some important tem- 
poral patterns, such as excitation followed by inhibition and rebound excitation. Initial physiological results in the amphibian optic tectum (Ingle, 1973, 1975, 1976a, b) support these assumptions.

The SP cell also receives its input from the glomerulus, and we propose that its function is the integration of the general state of activity of the column for the purpose of determining the proper time(s) for vertical recruitment of excitation in order to produce a response in the efferent cell of the column. In this way, the SP samples the excitatory interaction between the glomerulus and LP cell under the inhibitory effect of the stellate neuron.

We propose that the principal efferent of the column is the pyramidal cell (PY), which receives afferents from the LP and SP cells and acts as an output integrator for the activity of the column as a whole. The output of this cell could go to the spinal cord or reticular formation of the animal to yield motor output or could send axons to the thalamus which would establish tectal-thalamic loops.

It has been suggested (Ewert, 1970, 1976) that the thalamus and pretectum exert an inhibitory effect over the tectal activity. Fibers, possibly diencephalic in origin, have been found to form synapses in three areas of the tectum (Székely and Lázár, 1976): (1) around and within the glomerulus, where they may act through presynaptic inhibition; (2) in the intervening zone between glomeruli, where they probably exert postsynaptic inhibitory effects over the SP, LP, and PY dendrites; and (3) in some of the stellate cells, where they are likely to produce postsynaptic excitation. We modeled the effect of different paths for such inhibition.

\section{Computer Simulation}

To understand the general behavior of the column and the different hypotheses that can be postulated to underlie the observed results, we used simulation to study the following aspects of the model:

1. Activity of the PY cell when the stimulus is presented for different intervals of time and the optic fibers excite only the glomerulus.

2. Facilitation of the PY response when two stimuli are presented serially and the optic fibers project only to the glomerulus. In this section, we also study the behavior of the tectal cells in order to reproduce the observed physiological results.

3. Facilitation of the PY response when two stimuli are presented serially and the optic fibers project to the glomerulus and LP, SP, and PY cells. In this section, we also study the PY response for different durations of stimulus presentation.

4. Control of PY facilitation by the diencephalic fibers: $(a)$ inhibitory effects on the glomerulus; $(b)$ inhibitory effects on LP, SP, and PY cells; and (c) excitatory and inhibitory effects on the SN.

The results obtained with these models will provide us with specific hypotheses that could be tested experimentally and that could help to narrow the number of alternative models to explain the behavior.

The results of the simulation are shown in two ways: through the simulated behavior of each of the cells considered in the model and through graphs showing the sensitivity of response to variation of different parameters of stimulation. In the first case, we have simulated $5 \mathrm{sec}$ of real time to reproduce the behavioral and physiological results. We show diagrammatically the response of cells after the membrane potential has reached the threshold through the generation of spikes. We do not model the time course of spike generation. The spikes in the figures are a graphical convention designed to aid understanding of the behavior of the model; the way the model behaves when the membrane potential reaches threshold is explained in the appendix.

An important feature of our modeling methodology is that it lets us experiment with different connectivities. In the next two sections, the tectal column is described as a controller of the output through its general state of excitation, with the optic input arriving only at the glomeruli so that the activity of the PY is controlled by the LP and SP neurons. Next, we examine the postulate that the tectal column acts as a modulator of the PY response. In this model, the PY cell receives optic afferents directly so that its response is dependent on the conjoint activity of LP and SP cells and the stimulus. We shall argue that the latter version of the model represents observed behavior more realistically.

$P Y$ response to duration of presentation of the stimulus if the optic fibers arrive only at the glomeruli. Figure $2 a$ shows the neural response of tectal cells when a brief $(0.5-\mathrm{sec})$ stimulus is presented. Notice that the LP cells give a short response when the stimulus is presented and then a delayed burst of activity, reproducing the observed physiological results of tectal cells described by Ingle (1975). The SP neuron gives a short delayed response after the stimulus has disappeared, reproducing the physiological behavior of tectal cells. If we increase the period of presentation of the stimulus, then the PY cell, the efferent neuron of the model column, gives a response (see Fig. 2b). We consider the PY response to code the location and speed of prey-orienting behavior (Ewert, 1976; Lara and Arbib, 1982). The behavior of PY in Figure 2, $a$ and $b$ reproduces the observed behavior of amphibia when the period of presentation of a preystimulus is increased.

$P Y$ facilitation when two stimuli are presented serially and the optic fibers arrive only at the glomeruli. Figure $2 c$ shows the temporal pattern of response of the tectal cells during facilitation of prey-catching activity. When the stimulus is presented, the glomerulus produces a long EPSP. This potential makes the LP respond which, in turn, excites both the SN, which produces a long lasting hyperpolarization on LP and SP cells, and the glomerulus, which maintains its state of excitation. When the LP is released from the inhibitory effect of the $\mathrm{SN}$, it produces a new response because the glomerulus is still active, repeating this cycle several times until it finally decays. The SP cell, in the meantime, is integrating the state of activity of the glomerulus and the LP and SN cells until its membrane voltage reaches its threshold value which generates a response. The activity of the SP excites the LP cell, the glomerulus, and the $\mathrm{PY}$, increasing, in this way, the general state of excita- 

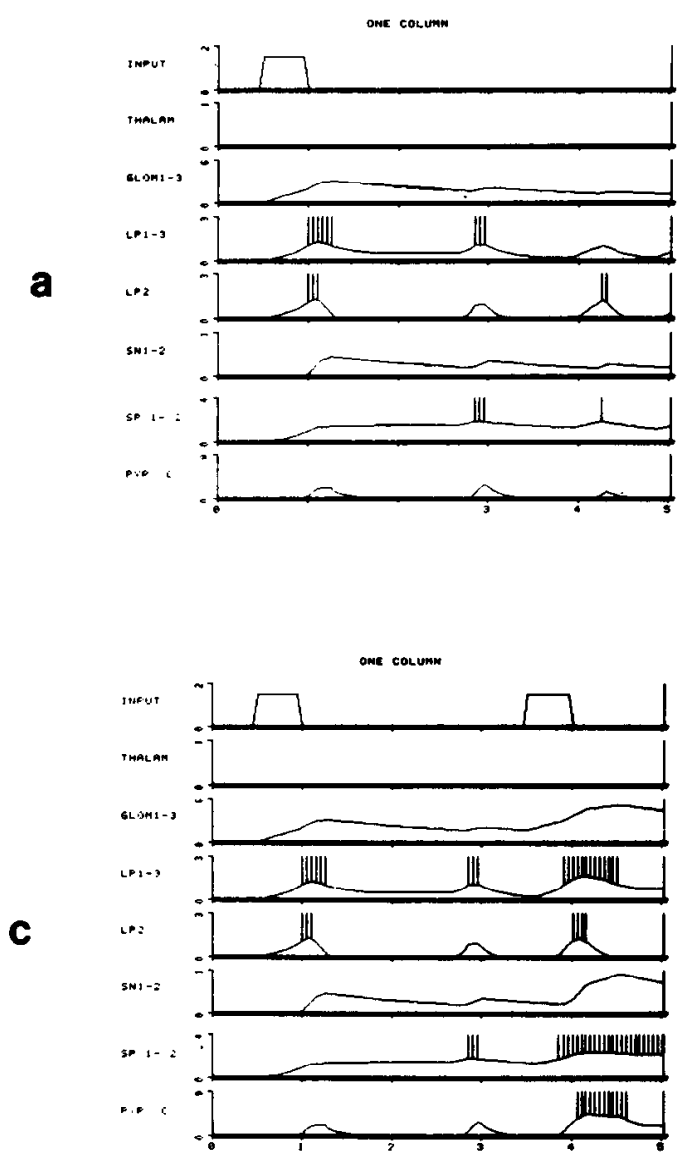
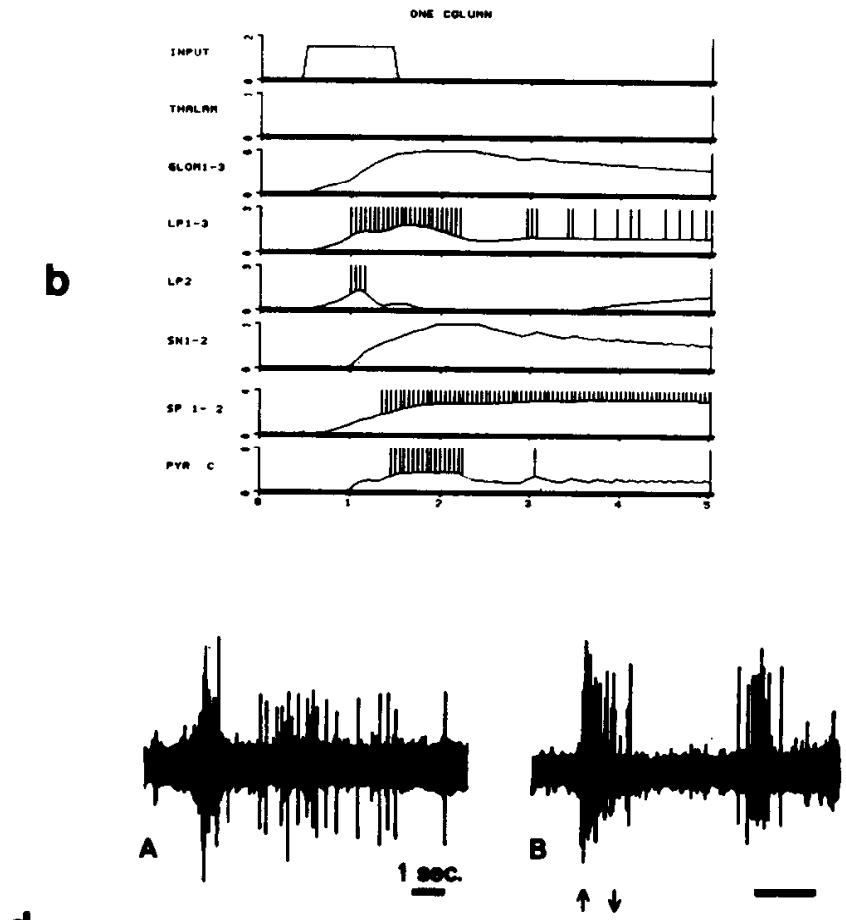

d
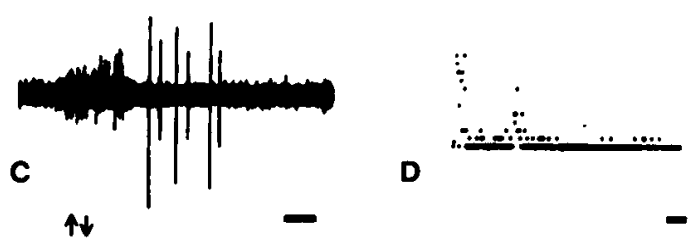

Figure 2. $a$, Computer simulation of the response of tectal cells when a brief stimulus is presented. (The frequency of the spikes are a graphical convention. The spikes are drawn simply to highlight when the membrane potential of a cell is above threshold.) The onset of the stimulus produces a long lasting depolarization in the glomerulus which then fires the large pear-shaped cell (LP). This neuron, in turn, sends recurrent axons to the glomerulus and the stellate cell (SN) which acts as the inhibitory neuron in the column. When the inhibitory effect of SN releases the LP cell, a rebounding excitation occurs. The small pear-shaped cell is integrating the activity of glomerulus and LP and SN neurons to give a delayed short response. $b$, If, in the above situation, we present a stimulus of longer duration, then we show that now the pyramidal neuron fires. In $c$, we show that, when a second stimulus of the "subthreshold duration" used in $a$ is presented, the pyramidal cell (PY) responds. $d$, Physiological behavior of cells related to prey-catching facilitation. $A$ shows a brief class 2 burst followed by a delayed response of a tectal cell. $B$ shows the response of a tectal cell to a stimulus; it emits two trains of pulses, one following the presentation of the stimulus and another a few seconds later. $C$ shows a tectal neuron that produces a delayed response to the presentation of the stimulus. Finally, $D$ shows the poststimulus histogram of a tectal cell showing a delayed peak at 3 to 4 sec. (From Ingle, 1975; used with permission from American Association for the Advancement of Science.) GLOM, glomerulus; PYR, pyramidal cell; $T H A L A M$, thalamus.

bility of the column. This figure shows how the model reproduces the behavior of the tectal cells described by Ingle (1975) (see Fig. 2d), which are related to preycatching facilitation. The LP cell reproduces the behavior of the neuron which responds to the onset of the stimulus and then exhibits a period of silence and finally a rebound activity that lasts for several seconds (Fig. $2 d, A$ and $B$ ), while the behavior of the SP simulates the activity of the neuron which does not respond when the stimulus is present but gives a short delayed response (Fig $2 d C$ ). Finally, the behavior of the PY cell, considered to be responsible for prey-catching activity, reproduces the behavioral results, because a response is given only to the second presentation of the short stimulus.

Facilitation of $P Y$ activity when stimuli are presented serially and the optic fibers arrive at the glomerulus and the LP, SP, and PY cells. Székely and Lázár (1976) have observed that the optic afferents to the tectum arrive mainly at the glomeruli, but there are also many synapses in the intervening zone between and somewhat lower than glomeruli arriving at light dendrites, probably of LP, SP, and PY cells. For this reason we also have studied the behavior of the tectal column so modified that optic input arrives at the LP, SP, and PY cells as well as the glomerulus. In this case, we postulate that the tectal column acts rather as a modulator than as a controller of the PY behavior. According to this, the PY responds only if the stimulus is present and if the column is in a state of hyperexcitation through the behavior of the LP and SP cells. Figure $3 a$ shows the PY response when stimuli are presented for different intervals. This figure shows that PY activity increases with the period of stimulation. Figure $3 b$ shows the temporal pattern followed by the facilitation effect when a stimulus is 


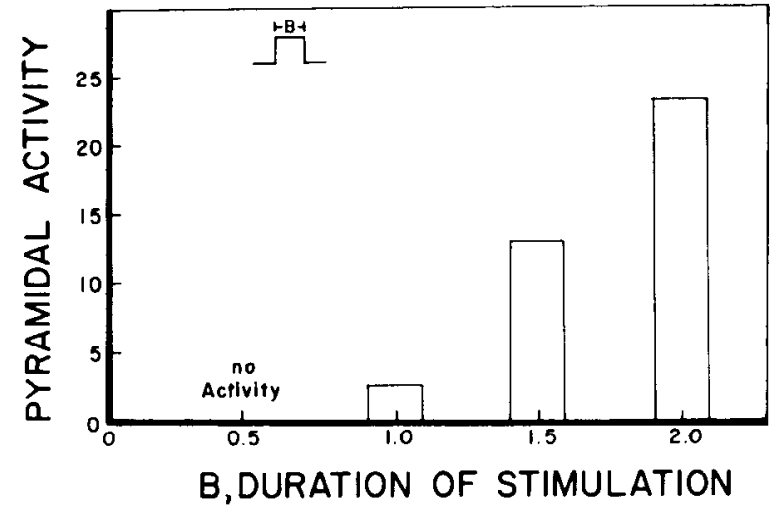

a

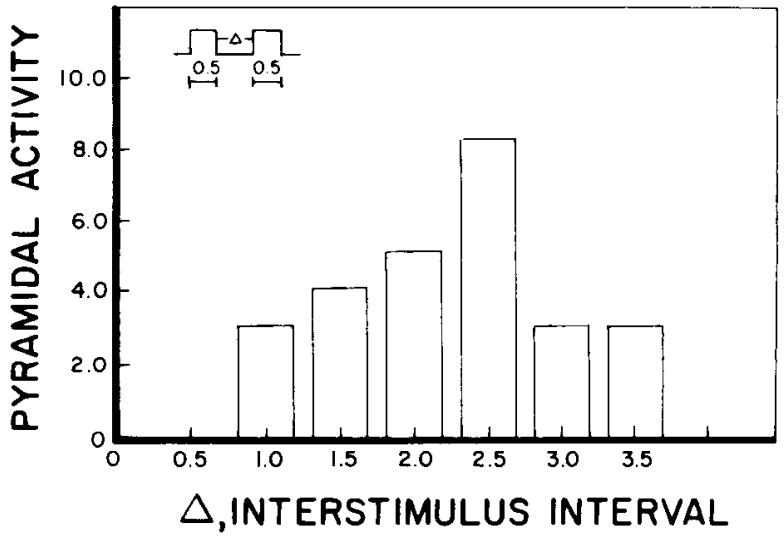

b

Figure 3. $a$, Computer simulation of the PY behavior when stimuli are presented for different intervals. The graph shows that the longer the presentation of the stimulus, the larger the PY response. $b$, Computer simulation of the temporal pattern of the facilitation process after the presentation of a brief stimulus. Each bar shows the duration of PY activity if the two stimuli of 0.5 sec duration are presented, with the interstimulus interval given below each bar. The graph shows that the maximum facilitation is presented $2.5 \mathrm{sec}$ after the presentation of the first stimulus.

applied. It can be seen that the facilitation reaches its maximum after a short period $(2.5 \mathrm{sec})$ and then decays slowly with sporadic rebounding facilitation.

In the structure described in the two preceding sections, the PY response is present long after the stimulus has disappeared, while for the structure described here simulation shows that the response is present only when the stimulus is present or has just disappeared. (For the details of this and further simulations, the reader may consult Lara y Zavala, 1982, section II.3.) We postulate that the activity of PY cells, in combination with pretectal neurons, plays a role in the location and intensity of the orienting response to a given stimulus. For this reason, we consider that the second model (optic fibers innervating the pyramidal cells, inter alia) better reproduces the behavioral results, because it gives more precise information about the actual site of the stimulus. In the next subsections, we will test the behavior of this second model.

It is also interesting to note that both models present phases of excitation and inhibition during the facilitatory period, which indicates that the speed of the response also will be modified, depending on which of these phases the column happens to be in during the presentation of the second stimulus.

Facilitation of $P Y$ activity and diencephalic inhibition. Anatomical studies of the tectum (Székely and Lázár, 1976; Trachtenberg and Ingle, 1974; Scalia, 1976) have shown that afferent fibers distinct from the optic ierminals arrive at the tectum. It is also well known that diencephalic terminals project to the tectum, suggesting that the fibers observed in Golgi studies come from these regions. These fibers have been found in the following sites: (1) the periphery of and within the glomerulus; (2) in the zone intervening between and somewhat below the glomeruli to the dendrites of LP, SP, and PY cells; and (3) the soma of the stellate neuron. We have simulated the possible inhibitory effects of diencephalic terminals on the facilitation of prey-catching activity. In this way, we can propose three possible ways in which diencephalic terminals could affect the facilitatory behavior of the column: (1) controlling the expression of facilitation, (2) erasure of the facilitation, and (3) suppression of facilitation.

We activated the diencephalic fibers after the presentation of the first stimulus and then studied the effects of the inhibitory action on the PY response when the second stimulus appeared. We report (but do not show) the time course of the cellular response for different configurations of the diencephalic terminals. Figure 4 shows the weights of the diencephalic terminals required to block facilitation.

Figure $4 A$ shows that, when the inhibitory action is over the dendrites of the glomerulus, it suppresses the excitatory dendrodendritic activity. In this condition, the facilitatory state of the column is erased, because the activity of the glomerulus, which is responsible for oscillatory behavior, has been suppressed.

As illustrated in Figure $4 B$, when the optic afferents are inhibited presynaptically by diencephalic terminals, the response of the PY cell decreases with increasing inhibition. However, in this situation, the state of excitation of the column is not affected directly.

In Figure $4 C$, when the inhibitory action is exerted on the dendrites of LP, SP, and PY cells, the facilitation decreases with increasing inhibition as in the above cases. The main difference here is that the inhibitory effects over the LP and SP cells prevent the continuation of the facilitatory effects, regulating, in this way, the development of the change. The rebounding excitation of LP cells and the SP activity are delayed, and, as a consequence of the thalamic inhibition, the PY neuron does not respond.

As depicted in Figure $4 D$, if the diencephalic terminals are simulated as excitatory, serving to control the inhibitory effect of the SN cell over LP and SP cells, the 

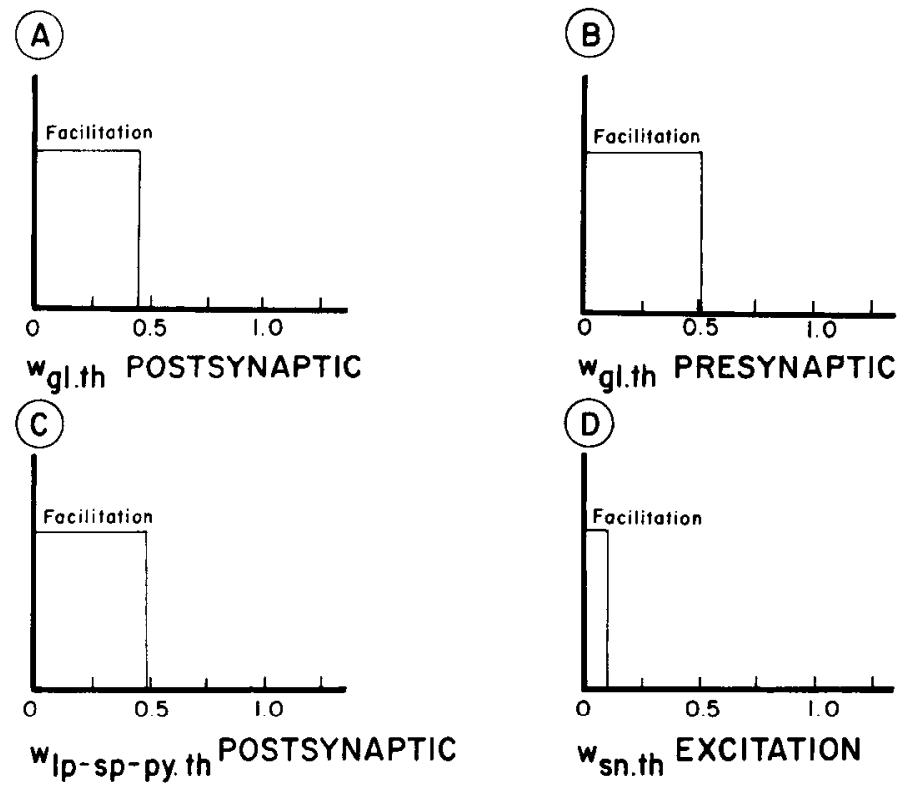

Figure 4. Sensitivity analysis of the effect of diencephalic inhibition over tectal facilitation in different zones. For low levels of inhibition, the facilitatory action of the tectal column is preserved. Above a critical value of the synaptic weight $w_{x \cdot t h}$ from thalamus to element $x$ of the tectal column, facilitation is suppressed. In $A, B$, and $C$, diencephalic fibers are posited to inhibit excitatory elements of the column. In $D$, the fibers are posited to excite stellate neurons, the inhibitory elements of the column. In each case, we show the range of synaptic weights insufficient to suppress facilitation. $A$, Postsynaptic inhibition to the glomerulus $(g l) ; B$, presynaptic inhibition to the optic input; $C$, postsynaptic inhibition to the LP, $\mathrm{SP}$, and PY cells; $D$, postsynaptic excitation to the SN. It can be seen that the strongest effect is produced through the SN neuron.

effective inhibitory action of these fibers is stronger than in the above cases, preventing the facilitation effect with small values of excitation. In this condition, as in that presented in Figure $4 C$, the diencephalon controls the development of the change. (If the diencephalic terminals are simulated as inhibiting the $\mathrm{SN}$, thus suppressing the inhibitory effect of $\mathrm{SN}$, all tectal cells respond in a paroxysmal manner.)

Change of architecture. One of the main goals in simulating the visuomotor system of amphibia is to present alternative models that reproduce the observed behavioral and physiological results and to propose specific experimental questions that will assist us in determining which of the models is closest to "reality." For this reason, the following change of architecture in the tectal column was tested to see if it also could reproduce the physiological and behavioral results observed by Ewert (1976) and Ingle (1976). 'This change of architecture was motivated by anatomical evidence provided by Székely and Lázár (1976) which indicates that the interaction between LP and SP cells could form a loop independent of that involving the glomerulus. Moreover, these authors have noted that SP cells do not have the same type of dendritic trees as LP cells, suggesting that they may have partially independent sources of excitation. For these reasons, we briefly studied a modified tectal column where the glomerulus receives the optic afferents and recurrent axons and contains the LP cell dendrites, where the LP and SP cells form an independent loop, wherein the SP cell is excited by the LP cell and the SP cell provides feedback in an excitatory loop to the LP cell, and where both cells are inhibited by the SN. The behavior of this model is shown in Figure 5, where it can be seen that SP and LP behavior are simultaneous, with both being similar to the behavior of LP neurons in the above models; any possibility of reverberatory activity between these cells is precluded by the inhibitory action of SN. In this case, the integrative activity proposed for the SP cell is lost and the physiological and behavioral results obtained by Ingle (1975) are not reproduced.

\section{Discussion}

The present model reproduces the facilitation of preycatching behavior when a brief stimulus, that initially does not produce a response, is presented for a second time. The model is based on anatomical studies of the optic tectum, physiological studies of tectal cells, and the behavioral results observed in prey-catching facilitation. Other assumptions considered in the model were taken from the results obtained in other brain regions that have similar structures and that present similar physiological behavior. For this reason, this model can be used to study the possible mechanisms responsible for oscillatory activity found in different regions, where periods of excitation,

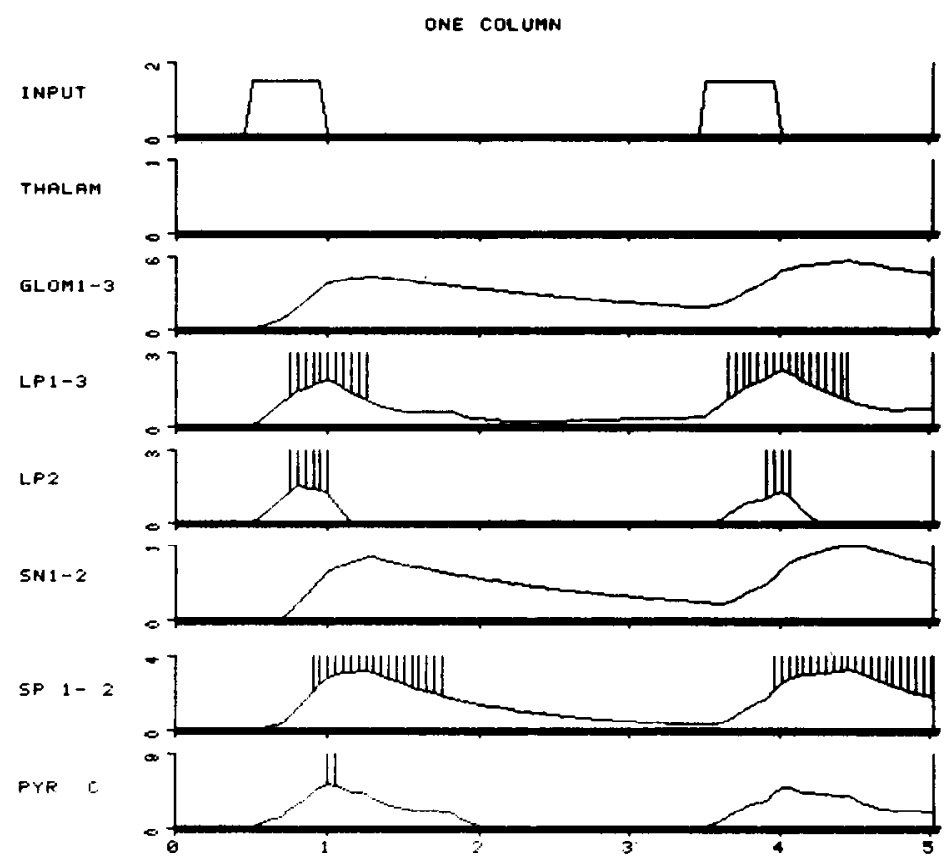

Figure 5. Computer simulation of an alternative architecture of the tectum in which the SP neuron now is activated only by LP neurons, forming a loop with positive feedback, but without receiving any optic input from the glomerulus. Notice that, during double presentation of a stimulus, the LP and SP cells respond simultaneously so that the dissociated behavior of these neurons is not present. This leads to rejection of the alternative. GLOM, glomerulus; PYR, pyramidal cells; $T H A L A M$, thalamus. 
inhibition, and rebounding excitation have been indicated. (For a sensitivity analysis of a reduced version of the present model, the reader may consult Lara y Zavala, 1982, section II.3.6.)

The correlation found by Fwert (1970) and Ingle (1973) between tectal cells and behavior is suggestive but by no means conclusive. It is important that a formal causal relationship be found and that the behavior of these neurons be correlated with other cells in the tectum within and outside of the postulated column. For example, Ingle's (1973) recordings were obtained in the superficial layers of the tectum, where SP cells are found, but the tectal efferents are located in deeper layers, thus indicating that a close relationship may exist between these two group of neurons. The present model proposes the following hypothesis that could be tested experimentally:

1. The input produces a long lasting depolarization in the glomerulus.

2. The initial response is produced by cells located in layer 6 and is silenced by the inhibitory effect of neurons located in layer 9 . These cells, which Ingle (1973) found rarely in layer 8 , should appear more frequently if the electrode goes deeper.

3. The combined effect of the long lasting depolarization and the inhibition produces the observed physiological behavior of excitation, silence, and rebounding excitation.

4. The delayed response is produced by the integration of the glomerulus and LP and SN neurons in the SP cell located in layer 8.

5. The efferent cell of the column is activated only if the state of excitation of the column has been increased as measured by the activity of tectal neurons.

6. Diencephalic terminals may control the state of excitation of the tectum in several ways, depending on the site of stimulation. The model indicates that excitation of the SN has a stronger effect on the general state of excitation of the tectum than inhibition at LP, SP, and PY cells or the glomerulus. When thalamic fibers arrive at the tectal dendrites, overt facilitation is suppressed, but long lasting depolarization of the glomerulus is still present. When diencephalic fibers directly inhibit the glomerulus, the facilitatory effect is disrupted completely. These results suggest that the interaction between the thalamus and tectum can play different roles and may have different temporal consequences. Each of these postulates could be tested following the same paradigm that we used in our simulation: presenting a brief stimulus, then exciting the thalamus, and observing the consequences when the second stimulus is presented. When the relationship of tectal cells has been studied, then the different effects of different thalamic regions can be studied.

The present model considers only the effect of ganglion cells of types 1 and 2, but the tectum also receives excitation from ganglion cells of types 3 and 4 . The latter neurons have been associated with avoidance behavior, indicating that the tectum may play a role in this activity through columnar structures similar to the ones proposed in this paper, and with close interactions with the thalamus. The study of the possible role of the tectum in avoidance behavior and its relationship with the orienting response still deserves more experimental and theoretical work.

\section{Appendix: Mathematical Description of the Column}

We represent the behavior of tectal neurons by a system of simultaneous differential equations which permit us to model the local (somatic membrane) potential, the threshold function, and the action potential of the cell. Due to the lack of adequate physiological information regarding the values of membrane constants for the different cells, we will use as approximate values the membrane constants of cells for which this parameter has been determined (Eccles, 1973).

The mathematical model of the tectal column is defined in terms of the different cells described anatomically for this structure. As we have mentioned, each column comprises three glomeruli, three large pearshaped cells, two small pear-shaped cells, two stellate neurons (only one of which is shown in Fig. 1), and one pyramidal cell. Each glomerulus contains optic fibers, dendrites and recurrent axons of $L P$ and SP cells, and diencephalic terminals. We have treated the firing rate of $\mathrm{SN}$ as proportional to its membrane potential (above a threshold) because it seems (Székely and Lázár, 1976) that SN can exert increasingly widespread effects as its state of excitation increases. The inputs to the tectal column are the optic and diencephalic afferents, while the response of the column is given by the large pyramidal cell. In the present paper, we do not model retinal response to the optic input, but simply provide each neuron with an input which encodes the physiologically appropriate stimulus. The tectal column is shown in Figure 1.

We associate with each element a membrane potential (denoted in lower case) and, with the exception of the glomerulus, a firing rate (denoted in upper case).

The fundamental equation describing the dynamics of each membrane potential $m(t)$ will be of the form

$$
\tau \dot{m}(t)=m(t)+I(t)
$$

where $\tau$ is the membrane constant and $I(t)$ represents the weighted sum of excitatory and inhibitory inputs.

The firing rate will be related to the membrane potential by a transfer function, $F(m-\theta)$, where $\theta$ is a suitable threshold value, and $F$ may be of either the form

$$
f(x)= \begin{cases}1 & \text { if } x>0 \\ 0 & \text { if not }\end{cases}
$$

or

$$
h(x)= \begin{cases}x & \text { if } x>0 \\ 0 & \text { if not }\end{cases}
$$

Our specific choices are shown in Table I.

Glomerulus. The glomerulus is considered to be a functional unit which receives as inputs the optic fibers and the recurrent axons of LP and SP cells. We have simulated the dendrodendritic activity of this structure 
TABLE I

Threshold functions for cell firing

\begin{tabular}{lcc}
\hline \multicolumn{1}{c}{ Element } & Membrane Potential & Firing Rate \\
\hline Glomerulus & $g l$ & \\
Large pear-shaped cell & $l p$ & $L P=f(l p-1.0)$ \\
Small pear-shaped cell & $s p$ & $S P=f(s p-2.0)$ \\
Stellate neuron & $s n$ & $S N=h(s n-0.2)$ \\
Thalamic input & $t h$ & $T H=h(t h)$ \\
Pyramidal cell & $p y$ & $P Y=h(p y-0.8)$ \\
\hline
\end{tabular}

simply by the decay constant of the state of activity of the glomerulus.

We use $g l_{i}(t)(i=1,2,3)$ to represent the membrane potential of the $i$ th glomerulus of the tectal column at time $t$. The basic equation for the dynamics of each $g l_{t}(t)$ is

$$
\tau_{g l} g l_{i}(t)=-k_{1} g l_{i}(t)+s \cdot u+I_{i}(t)
$$

where $\tau_{g l}=k_{1}=0.5$ so that $\left(\tau_{g l} / k_{1}\right)$ equals 1.0 , is the time constant of the glomerulus potential, and is chosen to simulate the excitatory dendrodendritic synapses that maintain a long EPSP (Shepherd, 1970) which is responsible for the rebound excitation after inhibition has been inactivated.

$u$ is the optic input, while $s$ is a habituation factor. We do not consider habituation in the present study and thus take $s$ to be 1 .

$I_{i}(t)$ is the recurrent input from the LP and SP cells. As shown in Figure 1, it takes the form

$$
\begin{aligned}
& I_{1}(t)=w_{g l \cdot s p} S P_{1}(t)+w_{g l \cdot l p}\left(L P_{1}(t)+L P_{2}(t)\right) \\
& I_{2}(t)=w_{g l \cdot s p}\left(S P_{1}(t)+S P_{2}(t)\right)+w_{g l \cdot l p}\left(L P_{1}(t)+L P_{2}(t)+L P_{3}(t)\right) \\
& I_{3}(t)=w_{g l \cdot s p} S P_{2}(t)+w_{g l \cdot s p}\left(L P_{2}(t)+L P_{3}(t)\right)
\end{aligned}
$$

where the different values of $w$, the weighting factors, are given in Table II. These values indicate that the recurrent axons of the LP cells, $w_{g l . l p}$, have a stronger effect over the glomerulus (GL) than those of the SP cells, $w_{g l . s p}$. This choice is made because, when the latter are active, the general level of activity of the column is very strong and the recurrent effect could result in unstable behavior (Lara y Zavala, 1982).

Stellate interneurons. We modeled each stellate cell as inhibiting the tectal column through feedback inhibition when it was stimulated by the LP cell. The SN also can be activated by the diencephalic terminals; for this reason, the dynamics of this cell are expressed as follows (see Fig. 3):

$$
\begin{aligned}
& \tau_{s n} \frac{d s n_{1}(t)}{d t}=-k_{2} s n_{1}(t)+w_{s n \cdot l p}\left(L P_{1}(t)+L P_{2}(t)\right)+w_{s n \cdot t h} T H(t) \\
& \tau_{s n} \frac{d s n_{2}(t)}{d t}=-k_{2} s n_{2}(t)+w_{s n \cdot l p}\left(L P_{2}(t)+L P_{3}(t)\right)+w_{s n \cdot t h} T H(t)
\end{aligned}
$$

where the membrane constant $\tau_{s n}=0.65, k_{2}=0.5$, and $\tau_{s n} / k_{2}=1.3$, which is chosen to simulate the long lasting hyperpolarization described for this type of cell in other brain regions. The weighting factors are defined in Table II. The effect $w_{s n . l p}$ of the LP over the SN cell was chosen to simulate the rapid inhibition that follows the excitation of the LP cells; $w_{s n \cdot t h}$ defines the effect of the thalamic input over the SN (explained in detail in our description of the general effect of diencephalic fibers over the tectum).

Large pear-shaped cells. LP cells receive excitatory input from the glomerulus and the SP cells and are inhibited by the SN and TH input. The equations defining the dynamics of this cell are expressed as follows:

$$
\tau_{l p} \frac{d l p_{i}(t)}{d t}=-l p_{i}(t)-w_{l p \cdot t h} T H(t)+J_{i}(t)+g l_{i}(t)
$$

where the intracolumnar inputs $J_{i}(t)(i=1,2,3)$ are given by:

$$
\begin{aligned}
& J_{1}(t)=w_{l p \cdot s p} S P_{1}(t)-w_{l p \cdot s n} S N_{1}(t) \\
& J_{2}(t)=w_{l p \cdot s p}\left(S P_{1}(t)+S P_{2}(t)\right)-w_{l p \cdot s n}\left(S N_{1}(t)+S N_{2}(t)\right) \\
& J_{3}(t)=w_{l p \cdot s n} S P_{2}(t)-w_{l p \cdot s n} S N_{2}(t)
\end{aligned}
$$

where $\tau_{l p}=0.3$ is the membrane constant defined to achieve the desired time course of response in the LP cells to the afferent stimulus from the glomerulus and the optic input. The $g l_{i}(t)$ values represent the transmission of excitation from the glomeruli to the LP cells; $w$ values are the weighting factors defined in Table II, where $w_{l p . s p}$ has been chosen to simulate the recruitment of cells produced by the SP neuron, $w_{l p \cdot s n}$ was chosen to simulate the strong long lasting hyperpolarization produced by the SN over the LP cell to control the general state of excitation of the column, and $w_{l p . t h}$ is the weight factor of the thalamic input over the LP neuron.

Small pear-shaped cells. As indicated above, the SP cells integrate the state of activity of the glomeruli and of the SN cells. The SP cells also receive an inhibitory input from the thalamus and from the SN neurons. The equations that define their behavior are expressed as follows:

$$
\begin{aligned}
& \tau_{s p} \frac{d s p_{1}(t)}{d t}=-s p_{1}(t)+\left(g l_{1}(t)+g l_{1}(t)\right)-w_{s p \cdot s n} S N_{1}(t)-w_{s p \cdot t h} T H(t) \\
& \tau_{s p} \frac{d s p_{2}(t)}{d t}=-s p_{2}(t)+\left(g l_{2}(t)+g l_{3}(t)\right)-w_{s p \cdot s n} S N_{2}(t)-w_{s p \cdot t h} T H(t)
\end{aligned}
$$

where $\tau_{s p}=0.9$ is the membrane constant of SP. This membrane constant is slow, in comparison to the other cells, to simulate the long lasting integration proposed for this neuron. The weighting factors, $w$, are shown in Table II, where it can be seen that the inhibitory effect of the SN is very strong, controlling, in this way, the

\section{TABLE II \\ Weights}

The weights marked with an asterisk are given zero or non-zero values in different experiments.

\begin{tabular}{ll}
\hline Weighting Factors & \\
\hline$w_{g l \cdot t p}=1.0$ & LP to GL \\
$w_{g l \cdot s p}=0.1$ & SP to GL \\
$w_{l p \cdot s p}=0.8$ & SP to LP \\
$w_{l p \cdot s n}=8.0$ & SN to LP \\
$w_{l p \cdot t h}=\star$ & TH to LP \\
$w_{s p \cdot s n}=15.0$ & SN to SP \\
$w_{s p \cdot t h}=\star$ & TH to SP \\
$w_{s n \cdot l p}=1.0$ & LP to SN \\
$w_{s n \cdot t h}=\star$ & TH to SN \\
$w_{p y \cdot t p}=1.0$ & LP to PY \\
$w_{p y \cdot s p}=1.0$ & SP to PY \\
\hline
\end{tabular}


general state of excitation of the column. 'The threshold function $S N=h(s n-0.2)$ was chosen in order to ensure the stability of the behavior of the column through an inhibition proportional to the state of activity of the stellate cell, which is also in accordance with the role proposed for the SN by Szekely and Lazar (1976). The $\mathrm{SP}$ cells receive optic afferents through the glomeruli; we have proposed that they receive information from two different glomeruli to ensure the general recruitment of all tectal cells.

\section{References}

Arbib, M. A., and R. Lara (1982) A neural model of the interaction of tectal columns in prey-catching behavior. Biol. Cybern., in press.

Eccles, J. C. (1973) The Understanding of the Brain, McGrawHill, New York.

Ewert, J. P. (1970) Neural mechanisms of prey catching and avoidance behavior in the toad (Bufo bufo). Brain Behav. Evol. 3: 35-56.

Ewert, J. P. (1976) The visual system of the toad: Behavioral and physiological studies on a pattern recognition system. In The Amphibian Visual System, K. V. Fite, pp. 142-202, Academic Press, New York.

Hubel, D. H., and T. N. Wiesel (1963) Shape and arrangement of columns in cat's striate cortex. J. Physiol. (Lond.) 165: $559-568$

Ingle, D. (1973) Disinhibition of tectal neurons by pretectal lesions in the frog. Science 180: 442-444.

Ingle, D. (1975) Focal attention in the frog: Behavioral and physiological correlates. Science 188: 1033-1035.

Ingle, D. (1976a) Spatial vision in anurans. In The Amphibian Visual System, K. V. Fite, ed., pp. 119-140, Academic Press, New York.

Ingle, D. (1976b) Visual correlates of central visual functions in anurans. In Frog Neurobiology, R. Llinas and W. Precht, eds., pp. 435-451, Springer-Verlag, Berlin.

Kandel, E. R., and W. A. Spencer (1961) Electrophysiology of hippocampal neurons. II. After potentials and repetitive firing. J. Neurophysiol. 24: 243-259.

Kandel, E. R., W. A. Spencer, and F. J. Brinley, Jr. (1961) Electrophysiology of hippocampal neurons. I. Sequential invasion and synaptic organization. J. Neurophysiol. 24: 225242.

Lara, R., and M. A. Arbib (1982) A neural model of interaction between tectum and pretectum in prey selection. Cognition Brain Theory 5: in press.
Lara y Zavala, R. (1982) Neural models of the visuomotor system of amphibia. Ph.D. dissertation, University of Massachusetts at Amherst, Amherst, MA.

Mountcastle, V. B. (1957) Modality and topographic properties of single neurons of cat's somatic sensory cortex. J. Neurophysiol. 20: 408-434.

Mountcastle, V. B. (1978) An organizing principle for cerebral function: The unit module and the distributed system. In The Mindful Brain, G. M. Edelman and V. B. Mountcastle, eds., pp. 7-50, MIT Press, Cambridge, MA.

Purpura, P. D. (1970) Operations and processes in thalamic and synaptically related neural subsystems. In The Neurosciences: Second Study Program, F. O. Schmit, ed., pp. 458471, The Rockefeller University Press, New York.

Raisman, G., W. M. Cowan, and T. P. S. De Powell (1965) The extrinsic afferents commissural and association fibres of the hippocampus. Brain 88: 963-996.

Scalia, F. (1976) The optic pathways of the frog: Nuclear organization and connections. In Frog Neurobiology, R. Llinas and W. Precht, eds., pp. 386-406, Springer-Verlag, New York.

Scheibel, M. E., and A. B. Scheibel (1970) Elementary processes in selected thalamic and cortical subsystems-the structural substratum. In The Neurosciences: Second Study Program, F. O. Schmitt, ed., pp. 443-458, The Rockefeller University Press, New York.

Shepherd, G. M. (1970) The olfactory bulb as a simple cortical system: Experimental analysis and functional implications. In The Neurosciences: Second Study Program, F. O. Schmitt, ed., pp. 539-551, The Rockefeller University Press, New York.

Shepherd, G. M. (1974) The Synaptic Organization of the Brain: An Introduction, Oxford University Press, London.

Singer, W. (1977) Control of thalamic transmission by corticofugal ascending reticular pathways in the visual system. Physiol. Rev. 57: 386-417.

Székely, G., and E. Lázár (1976) Cellular and synaptic architecture of the optic tectum. In Frog Neurobiology, R. Llinas and W. Precht, eds., pp. 407-434, Springer-Verlag, New York.

Szentágothai, J. (1970) Glomerular synapses, complex synaptic arrangements, and their operational significance. In The Neurosciences: Second Study Program, F. O. Schmit, ed., pp. 427-443, The Rockefeller University Press, New York.

Szentágothai, J., and M. A. Arbib (1974) Conceptual models of neural organization. Neurosci. Res. Program Bull. 12: 310479.

Trachtenberg, M. C., and D. Ingle (1974) Thalamo-tectal projections in the frog. Brain Res. 79: 419-430. 